\title{
Can gravitation accelerate neutrinos?
}

\author{
Sergio A. Hojman* \\ Departamento de Ciencias, Facultad de Artes Liberales, \\ Facultad de Ingeniería y Ciencias, Universidad Adolfo Ibáñez, Santiago, Chile, \\ and Departamento de Física, Facultad de Ciencias, Universidad de Chile, Santiago, Chile, \\ and Centro de Recursos Educativos Avanzados, CREA, Santiago, Chile. \\ Felipe A. Asenjd讨 \\ Institute for Fusion Studies, The University of Texas at Austin, Austin, Texas 78712, USA.
}

\begin{abstract}
The Lagrangian equations of motion for massive spinning test particles (tops) moving on a gravitational background using General Relativity are presented. The paths followed by tops are nongeodesic. An exact solution for the motion of tops on a Schwarzschild background which allows for superluminal propagation of tops is studied. It is shown that the solution becomes relevant for particles with small masses, such as neutrinos. This general result is used to calculate the necessary condition to produce superluminal motion in part of the trajectory of a small mass particle in a weak gravitational field. The condition for superluminal motion establishes a relation between the mass, energy and total angular momentum of the particle.
\end{abstract}

PACS numbers: 04.20.Cv, 04.20.Jb, 04.90.+e, 14.60.St, 14.60.Lm

\section{INTRODUCTION}

The well known September 2011 OPERA neutrino experiment [1] has produced a myriad of articles trying to explain or understand, prove or disprove the experimental results for superluminal neutrino motion. One of the most important contributions to the understanding of the problem is the Cohen-Glashow work [2].

Most of articles are based on models which, by construction, ignore or neglect general relativistic effects. Furthermore, even though the general relativistic aspects of the dynamics are not considered, the arguments presented in these papers seem to tacitly assume that neutrinos follow geodesics in the presence of gravitational fields.

It has been known for quite some time $3-6]$ that spinning massive test particles (tops) follow non geodesic paths when moving on gravitational fields.

Even though a recent communication seems to refute the fact that the original OPERA experimental results do indeed describe superluminal propagation of neutrinos, we believe that is interesting to show that a consistent general relativistic model allows for the possibility of superluminal propagation of tops moving on gravitational backgrounds [5 7].

In this article we discuss an exact solution for the equations describing the motion of a top on a Schwarzschild background. A Lagrangian derivation for these non geodesic equations of motion obeyed by tops moving on a gravitational background was first obtained by Hojman [5, 6] (using a formalism by Hanson and Regge [8] developed for flat spacetime) which it is outlined in the next

\footnotetext{
*Electronic address: sergio.hojman@uai.cl
}

†Electronic address: faz@physics.utexas.edu section. We show that massive tops may reach superluminal velocities provided that their particle mass $m$, energy $E$ and total angular momentum magnitude $j$ of the orbit, satisfy a relation (which is explicitly derived in Section IV).

Non geodesic equations of motion for tops were first derived by Mathisson [3] and Papapetrou [4] as limiting cases of rotating fluids moving in gravitational fields. On the other hand, massless spinning particles do follow null geodesics as showed by Mashhoon [9] who used the Mathisson-Papapetrou formalism for his derivation.

Loosely speaking, in the presence of gravitational fields, massive spinning particles (such as neutrinos) follow non geodesic paths $[3-6]$ while photons move in null geodesics (in spite of their spinning nature) because they are massless [9].

Even though the Equivalence Principle is sometimes interpreted as stating that test particles in a gravitational field should follow geodesics, this interpretation is, strictly speaking, valid only for spinless point test particles. Extended particles are, in general, subject to tidal forces and follow, therefore, non geodesic paths.

In the Lagrangian formulation of the motion of tops, the velocity $u^{\mu}$ and the canonical momentum $P^{\mu}$ vectors are, in general, not parallel. For the motion of tops in the presence of electromagnetic and/or gravitational fields, the (square of the) mass $m^{2}\left(\equiv P^{\mu} P_{\mu}>0\right)$ is conserved implying that the momentum vector remains timelike along the motion. Nevertheless, the velocity vector may become spacelike [5, 8, 11, 12].

The proper treatment of the (subtle but crucial) lack of parallelism between velocity and momentum is best achieved with a Lagrangian formulation of the motion of tops, because otherwise the canonical momentum cannot be appropriately defined. Furthermore, the MathissonPapapetrou formulation gives rise to third order equations of motion, while the Lagrangian approach gives rise 
to second order ones [ $[, 10]$.

It turns out that for suitable choices of initial conditions this non geodesic motion can accelerate tops (and neutrinos) beyond the speed of light.

Theoretical results involving superluminal propagation of massive spinning particles and fields in interaction with electromagnetic or gravitational fields have been reported previously in the literature by Velo and Zwanziger [11], Hanson and Regge [8], Hojman [5] and Hojman and Regge [12], for instance, while experiments reporting hints of superluminal neutrino propagation can be found in Refs. [13, 14], among others, and other results reported in the MIT webpage on Superluminal Neutrinos (The Net Advance of Physics, http://web.mit.edu/redingtn/www/netadv).

\section{MOTION OF A RELATIVISTIC TOP ON A GRAVITATIONAL FIELD}

Consider a relativistic (spherical) top. Denote its position by a four vector $x^{\mu}$ while its orientation is defined by an orthonormal tetrad $e_{(\alpha)}{ }^{\mu}$. A gravitational field is described as usual in terms of the metric field $g_{\mu \nu}[\underline{5},[6]$. The tetrad vectors satisfy

$$
g_{\mu \nu} e_{(\alpha)}^{\mu} e_{(\beta)}^{\nu} \equiv \eta_{(\alpha \beta)},
$$

with $\eta_{(\alpha \beta)}\left(=\eta^{(\alpha \beta)}\right)$ given by

$$
\eta_{(\alpha \beta)} \equiv \operatorname{diag}(+1,-1,-1,-1) .
$$

and have, therefore, six independent components (consistent with the number of parameters of the Lorentz group).

The velocity vector $u^{\mu}$ is defined in terms of an arbitrary parameter $\lambda$ by

$$
u^{\mu} \equiv \frac{d x^{\mu}}{d \lambda} .
$$
is

Besides, the antisymmetric angular velocity tensor $\sigma^{\mu \nu}$

$$
\sigma^{\mu \nu} \equiv \eta^{(\alpha \beta)} e_{(\alpha)}{ }^{\mu} \frac{D e_{(\beta)}^{\nu}}{D \lambda}=-\sigma^{\nu \mu},
$$

where the (covariant) derivative $D e_{(\beta)}{ }^{\nu} / D \lambda$ is defined in terms of the Christoffel symbols $\Gamma_{\rho \tau}^{\nu}$, as usual, by

$$
\frac{D e_{(\beta)}}{D \lambda} \equiv \frac{d e_{(\beta)}}{d \lambda}+\Gamma_{\rho \tau}^{\nu} e_{(\beta)}^{\rho} u^{\tau} .
$$

Note that general covariance may be achieved unambiguously at the level of the Lagrangian formulation [5] because only first derivatives of the dynamical variables are used in the construction of the Lagrangian. If implemented using the equations of motion, terms proportional to the Riemann tensor may be missed, because (flat spacetime) second partial derivatives commute while (curved spacetime) second order covariant derivatives do not commute and their commutator is proportional to the Riemann tensor. If no Lagrangian theory for a system of special relativistic equations of motion is known, the introduction of gravitational interactions cannot be unambiguously implemented.

The Lagrangian $L=L\left(a_{1}, a_{2}, a_{3}, a_{4}\right)$ is constructed as an arbitrary function of four invariants $a_{1}, a_{2}, a_{3}, a_{4}$ such that the action $S=\int L d \lambda$, be $\lambda$-reparametrization invariant

$$
L\left(a_{1}, a_{2}, a_{3}, a_{4}\right)=\left(a_{1}\right)^{1 / 2} \mathcal{L}\left(a_{2} / a_{1}, a_{3} /\left(a_{1}\right)^{2}, a_{4} /\left(a_{1}\right)^{2}\right),
$$

(the speed of light $c$ is set equal to 1 , at this time), $\mathcal{L}$ is an arbitrary function of three variables and $a_{1} \equiv u^{\mu} u_{\mu}, a_{2} \equiv$ $\sigma^{\mu \nu} \sigma_{\mu \nu}=-\operatorname{tr}\left(\sigma^{2}\right), a_{3} \equiv u_{\alpha} \sigma^{\alpha \beta} \sigma_{\beta \gamma} u^{\gamma}, a_{4} \equiv \operatorname{det}(\sigma)$. Note that this expression seems to require $a_{1}$ to be positive. To be precise, the fact that $a_{1}$ be positive is not crucial in the formulation, as it will be proved later on [see Eq. (21)]. One could rewrite the expression for the Lagrangian as

$$
L\left(a_{1}, a_{2}, a_{3}, a_{4}\right)=\left(a_{2}\right)^{1 / 2} \mathcal{L}_{1}\left(a_{1} / a_{2}, a_{3} /\left(a_{2}\right)^{2}, a_{4} /\left(a_{2}\right)^{2}\right),
$$

for instance.

The conjugated momentum vector $P_{\mu}$ and antisymmetric spin tensor $S_{\mu \nu}$ are defined by

$$
P_{\mu} \equiv \frac{\partial L}{\partial u^{\mu}},
$$

$$
S_{\mu \nu} \equiv \frac{\partial L}{\partial \sigma^{\mu \nu}}=-S_{\nu \mu} .
$$

The equations of motion are obtained by considering the variation of the action $S$ with respect to (ten) independent variations $\delta x^{\mu}$ and (the covariant generalization of) $\delta \theta^{\mu \nu}$ defined by

$$
\delta \theta^{\mu \nu} \equiv \eta^{(\alpha \beta)} e_{(\alpha)}^{\mu} \delta e_{(\beta)}^{\nu}=-\delta \theta^{\nu \mu} .
$$

The (non geodesic) equations of motion turn out to be [5, 6]

$$
\frac{D P^{\mu}}{D \lambda}=-\frac{1}{2} R_{\nu \alpha \beta}^{\mu} u^{\nu} S^{\alpha \beta},
$$

and

$$
\frac{D S^{\mu \nu}}{D \lambda}=S^{\mu \lambda} \sigma_{\lambda}{ }^{\nu}-\sigma^{\mu \lambda} S_{\lambda}{ }^{\nu}=P^{\mu} u^{\nu}-u^{\mu} P^{\nu} .
$$

These results hold for arbitrary $\mathcal{L}$. The dynamical variables $P^{\mu}$ and $S^{\mu \nu}$ may be interpreted as the ten generators of the Poincaré group. In order to restrict the spin tensor to generate rotations only, the Tulczyjew constraint [15]

$$
S^{\mu \nu} P_{\nu}=0,
$$

is usually imposed [5, 8]. It turns out that both the top mass $m$ and its spin $J$ are conserved quantities (see Appendix A)

$$
m^{2} \equiv P^{\mu} P_{\mu}
$$




$$
J^{2} \equiv \frac{1}{2} S^{\mu \nu} S_{\mu \nu}
$$

Furthermore, if $\xi^{\mu}$ is a Killing vector, then

$$
C_{\xi} \equiv P^{\mu} \xi_{\mu}-\frac{1}{2} S^{\mu \nu} \xi_{\mu ; \nu}
$$

is a constant of motion [5, 6, 12].

The following gauge choices and "invariant relations" (defined in Appendix B)

$$
x^{0}=\lambda=t, \quad e_{(0)}^{\mu}=P^{\mu} / m,
$$

may be implemented [5, 6, 8] to fix the arbitrary parameter $\lambda$ and to restrict the (Lorentz transformations) six degrees of freedom of the tetrad to three dimensional rotations (for details, please see Appendix B). The previous choices satisfy condition (14)

$$
e_{(0)}{ }^{\mu} e_{(0)}=\frac{P^{\mu} P_{\mu}}{m^{2}}=\eta_{(00)}=1,
$$

and are consistent with constraint (13)

$$
\begin{aligned}
\frac{D\left(S^{\mu \nu} P_{\nu}\right)}{D \lambda} & =0 \\
& =\left(S^{\mu \lambda} \sigma_{\lambda}^{\nu}-\sigma^{\mu \lambda} S_{\lambda}^{\nu}\right) P_{\nu}+m S^{\mu \nu} \frac{D e_{(0)_{\nu}}}{D \lambda} \\
& =m S^{\mu \lambda} \sigma_{\lambda}^{\nu} e_{(0)_{\nu}}-m S^{\mu \nu} \sigma_{\nu \lambda} e_{(0)}{ }^{\lambda}=0(19)
\end{aligned}
$$

where we have used the fact that

$$
\frac{D e_{(\alpha)}^{\mu}}{D \lambda}=-\sigma_{\lambda}^{\mu} e_{(\alpha)}^{\lambda}
$$

for any of the tetrad vectors, according to the Eqs. (4), (11) and (2).

The consistency of the constraint (13) with the equations of motion (11) and (12) is guaranteed by making use of the arbitrariness of Lagrangian $\mathcal{L}\left(\right.$ or $\left.\mathcal{L}_{1}\right)$ in $(6)$ (or (77) by appropriately constructing it. As a matter of fact, the Lagrangian [8]

$$
\begin{aligned}
L & =\left(\frac{A a_{1}-B a_{2}}{2}\right. \\
& \left.+\frac{1}{2} \sqrt{\left(A a_{1}-B a_{2}\right)^{2}-8 B\left(A a_{3}-2 B a_{4}\right)}\right)^{1 / 2}
\end{aligned}
$$

gives rise to the equations of motion (11) and (12) and the Tulczyjew constraint (13) plus a Regge trajectory defined by $B m^{2}-A J^{2} / 2=A B$. Besides, it can be proved this Lagrangian is well defined for $a_{1} \leq 0$. Therefore, one may consider Lagrangian (21) as the starting point of this theory.

In order to prove that (21) is a well defined Lagrangian for any value of $a_{1}$, define

$$
D \equiv \frac{1}{2} \sqrt{\left(A a_{1}-B a_{2}\right)^{2}+16 B^{2} a_{4}-8 A B a_{3}}
$$

such that the Lagrangian (21) now becomes

$$
L=\left(\frac{A a_{1}-B a_{2}}{2}+D\right)^{1 / 2}
$$

In what follows we show that $D \geq\left(A a_{1}-B a_{2}\right) / 2$ which ensures that $L$ is real for any value of $a_{1}$ under very general assumptions.

First, we can see that $a_{4}$ is always non negative because the determinant of an antisymmetric matrix in an even dimensional space is a perfect square. The sign of $a_{3}$ can be found as follows. We have that

$$
a_{3} \equiv u_{\alpha} \sigma^{\alpha \beta} \sigma_{\beta \gamma} u^{\gamma}
$$

may be rewritten as

$$
a_{3}=-U_{\alpha} U^{\alpha}
$$

with

$$
U^{\alpha} \equiv \sigma^{\alpha \beta} u_{\beta}
$$

Consider the momentum vector $P^{\alpha}$ which is always timelike (due to mass conservation). We now show that $U_{\alpha}$ is a spacelike vector because it is orthogonal to $P^{\alpha}$. The orthogonality relation reads

$$
P^{\alpha} U_{\alpha}=P^{\alpha} \sigma_{\alpha}{ }^{\beta} u_{\beta}=\frac{D P^{\beta}}{D \lambda} u_{\beta}=0,
$$

where we have used (20) for $e_{(0)}^{\mu}=P^{\mu} / m$, and the fact that $u_{\beta}$ is orthogonal to $D P^{\beta} / D \lambda$ as it can be easily seen from (11). Therefore, $U_{\alpha} U^{\alpha}<0$, and then $a_{3}$ is positive.

It is enough to choose $A B<0$ to end the proof that the Lagrangian (21) is well defined irrespective of the sign of $a_{1}$. Even if $A B \geq 0$ there are regions where Lagrangian (21) is well defined for $a_{1}<0$, but for $A B<0$, it is well defined everywhere.

\section{EXACT SOLUTION}

The equatorial motion of a top in a Schwarzschild field background may be solved exactly. The Schwarzschild line element is $d s^{2}=g_{t t} d t^{2}+g_{r r} d r^{2}+g_{\theta \theta} d \theta^{2}+g_{\phi \phi} d \phi^{2}$, where $g_{t t}=c^{2}\left(1-2 r_{0} / r\right), g_{r r}=\left(1-2 r_{0} / r\right)^{-1}, g_{\theta \theta}=$ $-r^{2}, g_{\phi \phi}=-r^{2} \sin ^{2} \theta$, and $2 r_{0}$ is the Schwarzschild radius. From now on we reinsert explicitly $c$ in all the expressions. The general equations (11), (12) were written in Ref. [5] along with (13), (14), (15), (16) and (17) for the four Killing vectors of the Schwarzschild metric. In this article, we restrict ourselves to the motion in the plane defined by $\cos \theta=0$. If the top is initially in that plane and $\dot{\theta}=0$, then it remains in the equatorial plane [5], in which $\theta=\pi / 2$ and $P^{\theta}=0$. This reduction is possible due to the fact that the direction of angular momentum is conserved, so two of the four Killing vector conservation laws are used to restrict the motion to the equatorial plane. 
It is convenient to define the dimensionless parameter

$$
\eta=\frac{J^{2} r_{0}}{m^{2} c^{2} r^{3}}
$$

where $J=\hbar / 2$ is the top's spin (as well as the neutrino's spin). Thus, the set of equations (11)-117) (including the two remaining Killing vector conservation laws, energy $E$ and total angular momentum magnitude $j$ in addition to the conservation of mass and spin) may be solved exactly to yield [5]

$$
\begin{aligned}
& P_{\phi}=\frac{-j \pm E J /\left(m c^{2}\right)}{1-\eta}, \\
& P_{t}=\frac{E \mp j J r_{0} /\left(m r^{3}\right)}{1-\eta},
\end{aligned}
$$

and, from $P_{\mu} P^{\mu}=m^{2} c^{2}$, we get

$$
P^{r}= \pm\left[\frac{P_{t}^{2}}{c^{2}}-\left(\frac{P_{\phi}^{2}}{r^{2}}+m^{2} c^{2}\right)\left(1-\frac{2 r_{0}}{r}\right)\right]^{1 / 2}
$$

We can now solve for the velocities. To this end, we use two of the equations of motion (12). In the plane defined by $\theta=\pi / 2$, these equations become

$$
\begin{aligned}
\frac{D S^{t r}}{D \lambda} & =P^{t} \dot{r}-P^{r} \\
& =\frac{S^{\phi r} P_{\phi}}{P_{t}^{2}} \frac{D P_{t}}{D \lambda}-\frac{D S^{\phi r}}{D \lambda} \frac{P_{\phi}}{P_{t}}-\frac{S^{\phi r}}{P_{t}} \frac{D P_{\phi}}{D \lambda}
\end{aligned}
$$

and

$$
\begin{aligned}
\frac{D S^{t \phi}}{D \lambda} & =P^{t} \dot{\phi}-P^{\phi} \\
& =-\frac{S^{\phi r} P_{r}}{P_{t}^{2}} \frac{D P_{t}}{D \lambda}+\frac{D S^{\phi r}}{D \lambda} \frac{P_{r}}{P_{t}}+\frac{S^{\phi r}}{P_{t}} \frac{D P_{r}}{D \lambda}
\end{aligned}
$$

which may be solved for $\dot{r}$ and $\dot{\phi}$. To perform this task, we use the equations of motion (11) and (12), the relations between the spin and momentum [5]

$$
\begin{gathered}
S^{t r}=-\frac{S^{\phi r} P_{\phi}}{P_{t}}, \\
S^{t \phi}=\frac{S^{\phi r} P_{r}}{P_{t}},
\end{gathered}
$$

which are consequences of the constraint (13), and the condition

$$
\left(S^{\phi r}\right)^{2}=\frac{J^{2}\left(P_{t}\right)^{2}}{m^{2} r^{2}}
$$

which is derived from Eqs. (13), (14) and (15).
Taking these results into account, the velocities turn out to be

$$
\begin{gathered}
\dot{\phi}=\frac{c^{2}}{r^{2}}\left(1-\frac{2 r_{0}}{r}\right)\left(\frac{2 \eta+1}{\eta-1}\right)\left(\frac{P_{\phi}}{P_{t}}\right), \\
\dot{r}=c^{2}\left(1-\frac{2 r_{0}}{r}\right)\left(\frac{P^{r}}{P_{t}}\right) .
\end{gathered}
$$

Finally, we get

$$
\frac{d \phi}{d r}=\left(\frac{2 \eta+1}{\eta-1}\right)\left(\frac{P_{\phi}}{r^{2} P^{r}}\right) .
$$

It is worth noting that the three preceding expressions coincide with the usual results for geodesic motion when $J^{2}=0$ (and therefore $\eta=0$ ). Once the solutions are spelled out, we can find one of the main results of this work. From expressions (37) and (38), we find

$$
\left(\frac{d s}{c d t}\right)^{2}=\frac{g_{t t}}{c^{2}}+g_{r r}\left(\frac{\dot{r}}{c}\right)^{2}+g_{\phi \phi}\left(\frac{\dot{\phi}}{c}\right)^{2}=\frac{m^{2}}{\left(P^{t}\right)^{2}}(1-\Lambda),
$$

where $c^{2} P^{t}=\left(1-2 r_{0} / r\right)^{-1} P_{t}$, and we define the superluminal parameter $\Lambda$ as

$$
\Lambda=\frac{3 \eta(2+\eta)}{m^{2} c^{2} r^{2}(1-\eta)^{2}}\left(P_{\phi}\right)^{2}>0 .
$$

From (40) is straightforward to realize that it is possible that $d s^{2}<0$ for some of the solutions, at least in part of the top's trajectories. It is clear that the contribution of the $\Lambda$ parameter is important for small mass particles, such as neutrinos, because the $\Lambda$ dependence on the particle mass behaves as $m^{-4}$.

In the next section we study the trajectory of small mass particles under the approximation in which the top moves far away from the Schwarzschild horizon, i.e., for $r \gg r_{0}$. We find the conditions that the mass, energy and total angular momentum of the top must satisfy in order to produce superluminal motion in a segment of its trajectory.

\section{SUPERLUMINAL MOTION FOR $r \gg r_{0}$}

The main purpose of this section is to show that there are some particle trajectories such that $\Lambda<1$ in part of the path and $\Lambda>1$ in the rest of it. Knowing that $J=\hbar / 2$ is the spin of the top, superluminal behavior imposes a condition on the mass $m$, the total energy $E$, and the total angular momentum $j$.

We look for a solution such that the particle is moving at distances $r$ much larger than the Schwarzschild radius of the black hole, $r_{0} / r \ll 1$, i.e., we only consider the motion of the particle in a weak gravitational field. Besides, to focus in regimes in which $\Lambda$ is relevant, we restrict ourselves to the case of a small mass particle, such 
that its total energy is larger than its rest mass energy, $E \gg m c^{2}$. Both assumptions imply that $\eta \ll 1$.

In the weak field and small mass approximations, taking the upper signs in the preceding solutions (29) and (30), the momenta become $P_{\phi}=$ $(1+\eta)\left(-j+E J / m c^{2}\right), P_{t}=(1+\eta)\left(E-j J r_{0} / m r^{3}\right)$, and

$$
P^{r}= \pm\left[\frac{E^{2}}{c^{2}}-\frac{1}{r^{2}}\left(j-\frac{E J}{m c^{2}}\right)^{2}\right]^{1 / 2} .
$$

In order to study the particle motion, we assume that initially the particle approaches the central body from infinity and it remains always at a distance $r \gg r_{0}$. Thus, the orbit of the particle in the equatorial plane can be characterized by two values of $r$ in the trajectory, $r_{c}$ and $r_{R}$. The first one is the value of the orbit's radius in which the particle's velocity reaches the speed of light, i.e., the point in which $\Lambda=1$. The second one is the return point of the particle's orbit, and it is defined as the point when its radial momentum vanishes, $P^{r}=0$.

By imposing the condition $\Lambda=1$, it is straightforward to obtain that the value of $r_{c}$ is given by

$$
r_{c}=\left[\frac{6 J^{2} r_{0}}{m^{4} c^{4}}\left(j-\frac{E J}{m c^{2}}\right)^{2}\right]^{1 / 5} .
$$

On the other hand, we can calculate the return point for the particle's trajectory $r_{R}$ solving $P^{r}=0$ from (42). We get

$$
r_{R}=\frac{c j}{E}\left(1-\frac{J E}{j m c^{2}}\right) .
$$

The particle reaches the speed of light in $r_{c}$ (because $\Lambda\left(r_{c}\right)=1$ and therefore $d s^{2}=0$ ). However, to have superluminal motion after that point requires that $\Lambda\left(r_{R}\right)>1$ (implying $d s^{2}<0$ ). Using Eq. (44) in (41), this implies a condition that the mass, the energy and the total angular momentum of the particle must fulfill

$$
6 J^{2} E^{5} r_{0}>m^{4} c^{9}\left(j-\frac{E J}{m c^{2}}\right)^{3} .
$$

The above expression shows the relation that $m, E$ and $j$ satisfy to achieve superluminal motion. Notice that the condition cannot be met by spinless particles. Knowing the energy of the particle, it is possible to use (45) to estimate the mass and total angular momentum of the particle if superluminal motion is ever detected.

Interestingly, one can prove that condition (45) is equivalent to $r_{R}<r_{c}$. This means that when the particle has a "ballistic" trajectory around the black hole, it can always be superluminal in some part of its orbit, nearest to $2 r_{0}$, for appropriate $m, E$ and $j$. Clearly its speed will not be constant along the trajectory, because in the first part of the trip, the gravitational field speeds it up until it reaches $r_{R}$, slowing it down afterwards.

\section{CONCLUSIONS}

We present an exact solution to the non geodesic equations of motion in a Schwarzschild gravitational background that allows for superluminal propagation of massive spinning test particles. The superluminal motion depends strongly of the inverse of the mass of the particle. This is, of course, relevant for small mass particles such as neutrinos. We have shown that a consistent general relativistic theory which allows for superluminal propagation is possible.

Furthermore, the superluminal propagation effect presented here can be achieved in weak gravitational fields, as for example, in the surface of the Earth. In the presence of stronger gravitational fields (which can be easily found in astrophysical context) this effect will be much enhanced.

It is worthwhile mentioning that the aforementioned effect depends strongly on the fact that we deal with small mass particles, as the relevant $\Lambda$ parameter has an $m^{-4}$ dependence, and it is therefore very unlikely that superluminality could be detected for particles other than neutrinos.

Finally, we would like to mention that the equation of motion (11) can be rigorously generalized to include the gravitational self-force of the tops [16], showing that these corrections also modify the geodesic paths. However, the new forces are proportional to the particle mass and therefore their effects for small mass particles are negligible compared with those presented in this work.

\section{Appendix A}

The mass conservation law may be obtained as follows. Rewrite the velocity vector $u^{\nu}$ as

$$
\begin{aligned}
u^{\nu} & =\frac{1}{m^{2}}\left(P_{\mu} P^{\mu}\right) u^{\nu}+\frac{1}{m^{2}}\left(P_{\mu} u^{\mu}-P_{\mu} u^{\mu}\right) P^{\nu} \\
& =\frac{1}{m^{2}}\left(P^{\mu} u^{\nu}-P^{\nu} u^{\mu}\right) P_{\mu}+\frac{1}{m^{2}} P_{\mu} u^{\mu} P^{\nu} .
\end{aligned}
$$

We use the equation of motion for the spin tensor (12) to deal with the first term on the right hand side of the previous equation. Then

$$
\begin{aligned}
m^{2} u^{\nu} & =P_{\mu} \frac{D S^{\mu \nu}}{D \lambda}+P_{\mu} u^{\mu} P^{\nu}, \\
& =-\frac{D P_{\mu}}{D \lambda} S^{\mu \nu}+P_{\mu} u^{\mu} P^{\nu},
\end{aligned}
$$

where we have used the constraint $S^{\mu \nu} P_{\mu}=0$. We multiply by $D_{\lambda} P_{\nu}$, and due to the fact that $D_{\lambda} P_{\mu} S^{\mu \nu} D_{\lambda} P_{\nu} \equiv$ 0 , we find

$$
m^{2} u^{\nu} \frac{D P_{\nu}}{D \lambda}=P_{\mu} u^{\mu} P^{\nu} \frac{D P_{\nu}}{D \lambda} .
$$

However, using the equation of motion for $P_{\nu}$, we find that $u^{\nu} D_{\lambda} P_{\nu}=0$, and then we get the condition

$$
P^{\nu} \frac{D P_{\nu}}{D \lambda}=0
$$


which implies that $m^{2}$ is constant.

\section{Appendix B}

In this Appendix we show how to implement the choices

$$
e_{(0)}^{\mu}=P^{\mu} / m \text {. }
$$

using a different approach to the one presented in [8].

Let's start by defining $\bar{S}_{(\alpha \beta)}$ by

$$
\bar{S}_{(\alpha \beta)} \equiv e_{(\alpha) \mu} S^{\mu \nu} e_{(\beta) \nu} .
$$

It is a straightforward matter to realize that the six quantities $\bar{S}_{(\alpha \beta)}$ are constants of motion

$$
\frac{D \bar{S}_{(\alpha \beta)}}{D \lambda}=0,
$$

because of (12) and (20). We can now choose

$$
\bar{S}_{(0 i)}=0,
$$

as three initial conditions which are, of course, preserved in time because of (B3). Hanson and Regge call these conditions "invariant relations".

Conditions (B4) imply that the vector $e_{(0) \mu}$ is a null eigenvector of the spin matrix $S^{\mu \nu}$, i.e.,

$$
S^{\mu \nu} e_{(0) \nu}=0 .
$$

The spin matrix $S^{\mu \nu}$ has even rank (because it is antisymmetric). Due to the fact that $S^{\mu \nu} P_{\nu}=0$, its rank is not four, so it must be two (otherwise it would be zero, rendering it trivial). If its rank is two, it must have two null eigenvectors, which are the momentum vector $P^{\mu}$ and the Pauli Lubanski vector $W^{\mu} \equiv \frac{1}{2} \epsilon^{\mu \nu \alpha \beta} S_{\alpha \beta} P_{\nu}$, as one can easily prove (see also [8]).

Therefore, the vector $e_{(0)}{ }^{\mu}$ may be expressed as a linear combination of the momentum vector $P^{\mu}$ and the Pauli Lubanski vector $W^{\mu}$

$$
e_{(0)}^{\mu}=\rho \frac{P^{\mu}}{m}+\tau W^{\mu} .
$$

Hanson and Regge [8] construct the Hamiltonian theory of the top (pages 523 and following of reference [8]) using Dirac's method [17]. There they handle the Tulczyjew constraint $S^{\mu \nu} P_{\nu}=0$ by extracting its first class content $\Phi_{2}$ (in Dirac's terminology) with

$$
\Phi_{2} \equiv \frac{1}{2} \epsilon^{\mu \nu \alpha \beta} S_{\mu \nu} S_{\alpha \beta} \approx 0,
$$

where the sign " $\approx 0$ " is read "weakly equal to zero". This means that the constraint $\Phi_{2}$ vanishes, but its Poisson bracket relations with some dynamical variables are different from zero. Loosely speaking, Dirac's method provides one way to mend this contradiction by redefining the Poisson brackets relations. The new brackets are called Dirac brackets in his honor. The Dirac brackets of the constraints (with any dynamical variable) are identically zero.

Due to the fact that $\Phi_{2}$ is first class, it generates gauge transformations [17]. Therefore a gauge (associated to it) may be chosen. If the Poisson bracket of a dynamical variable $A$ with $\Phi_{2}$ is such that $\left[A, \Phi_{2}\right] \approx 0$, then $A$ is invariant under $\Phi_{2}$, i.e., is gauge invariant. So, in order to choose a gauge associated to $\Phi_{2}$ one needs to find a variable $B$ such that its Poisson bracket with $\Phi_{2}$ be different from zero.

Use (B6) to get $\rho$

$$
\rho=e_{(0)}{ }^{\mu} \frac{P_{\mu}}{m},
$$

and consider the Poisson bracket relations, equations (3.11) of reference [8]

$$
\left[e_{(\gamma)}^{\mu}, S^{\alpha \beta}\right]=e_{(\gamma)}^{\alpha} g^{\mu \beta}-e_{(\gamma)}^{\beta} g^{\mu \alpha},
$$

or

$$
\left[e_{(\gamma)}{ }^{\mu}, S_{\alpha \beta}\right]=e_{(\gamma) \alpha} \delta^{\mu}{ }_{\beta}-e_{(\gamma) \beta} \delta^{\mu}{ }_{\alpha},
$$

(it is perhaps worth mentioning that there is a change in notation, the role of Hanson and Regge's $\Lambda_{\mu}{ }^{\nu}$ matrix [8] is played by the tetrad vectors [5, [6] $e_{(\mu)}^{\nu}$ here). Now, it is straightforward to realize that $\left[\rho, \Phi_{2}\right]=4 e_{(0) \mu} W^{\mu} \neq 0$ (if $e_{(0) \mu} W^{\mu}=0$, the proof ends here).

Squaring (B6) one gets

$$
1=\rho^{2}-\tau^{2} m^{2} J^{2} .
$$

We may, therefore, choose the gauge

$$
\rho=1
$$

which means that $\tau=0$, thus ending the proof.

The same result may be achieved by computing $\left[\tau, \Phi_{2}\right]$ and choosing the gauge $\tau=0$.

\section{Acknowledgments}

F.A.A. thanks the CONICyT-Chile for a BecasChile Postdoctoral Fellowship.
[1] T. Adam et al., The OPERA Collaboration, arXiv:1109.4897 (2011).
[2] A. G. Cohen and S. L. Glashow, Phys. Rev. Lett. 107, 181803 (2011). 
[3] M. Mathisson, Acta Physica Polonica 6, 163 (1937); 6, 218 (1937).

[4] A. Papapetrou, Proc. Roy. Soc (London) 209A, 248 (1951).

[5] S. A. Hojman, Electromagnetic and Gravitational Interactions of a Spherical Relativistic Top, Ph.D. thesis, Princeton University, 1975 (unpublished).

[6] S. Hojman, Phys. Rev. D 18, 2741 (1978).

[7] R. Hojman and S. Hojman, Phys. Rev. D 15, 2724 (1977).

[8] A. J. Hanson and T. Regge, Annals of Physics 87, 498 (1974).

[9] B. Mashhoon, Annals of Physics 89, 254 (1975).

[10] S. N. Rasband, Phys. Rev. Lett. 30, 111 (1973).

[11] G. Velo and D. Zwanziger, Phys. Rev. 186, 1337 (1969);
188, 2218 (1969).

[12] S. Hojman and T. Regge, Studies in Mathematical Physics, Essays in Honor of Valentin Bargmann, Ed. E.H. Lieb, B. Simon and A.S. Wightman, Princeton University Press, pg. 195 (1976).

[13] G. Cacciapaglia, A. Deandrea and L. Panizzi, J. High Energy Particles 11, 137 (2011).

[14] S. Giani, AIP Conf. Proc. 458, 881 (1999).

[15] W. Tulczyjew, Acta Physica Polonica 18, 393 (1959).

[16] S. E. Gralla and R. M. Wald, Class. Quantum Grav. 25 205009 (2008); Erratum-ibid. 28159501 (2011).

[17] P. A. M. Dirac, Lectures in Quantum Mechanics, Belfer Graduate School of Science, Yeshiva University, New York (1964) 